\title{
Bidirectional solitons on water
}

\author{
Jin E. Zhang ${ }^{1}$ and Yishen $\mathrm{Li}^{2}$ \\ ${ }^{1}$ Department of FINA, Hong Kong University of Science and Technology, Clear Water Bay, Kowloon, Hong Kong \\ ${ }^{2}$ Department of Mathematics and Center of Nonlinear Science, University of Science and Technology of China, Hefei 230026, China
}

(Received 6 August 2002; published 24 January 2003)

\begin{abstract}
A theory of bidirectional solitons on water is developed by using an integrable Boussinesq surface-variable equation. We present an explicit transformation between the system and a member of the Ablowitz-KaupNewell-Segur system, and derive an exact multisoliton solution by using a Darboux transformation. The phase shifts and the maximum wave heights during the interaction are studied for two-soliton overtaking and head-on collisions. They agree with the Korteweg-de Vries solution for overtaking collision and the perturbation solution for head-on collision.
\end{abstract}

DOI: 10.1103/PhysRevE.67.016306

PACS number(s): 47.90.+a, 05.45.Yv, 02.30.Ik, 02.30.Jr

\section{INTRODUCTION}

Bidirectional solitary waves on water has been an interesting issue for many years, and enjoys an extensive literature $[1,2]$, partially because the model can be used to study the run-up of ocean waves such as tsunami waves on dykes and dams [3]. Existing literature solves the problem for an approximate solution by using a perturbation method on the Euler equation $[1,2]$.

In 1965, Zabusky and Kruskal introduced the concept of a soliton for the Korteweg-de Vries (KdV) equation [4]. Two years later, by using the inverse scattering method on the Schrödinger equation, Gardner et al. (GGKM) solved the $\mathrm{KdV}$ equation for exact $N$-soliton solutions [5], which can be used to model the interaction of unidirectional solitary waves on water. Their discovery establishes the mathematical foundation of the unidirectional water wave interaction. The KdV equation is the leading-order approximation of the Euler equation from a perturbation scheme under the assumption that the wave height is relatively small and the wavelength is relatively long compared with the water depth. It also assumes that the wave propagates in one direction, which is not a good assumption to model the reflection of water waves on a vertical wall. For reflection of water waves, we need a model that allows the bidirectional wave interactions, including head-on and overtaking collisions. As far as we know, a solid mathematical foundation of the bidirectional water wave interaction has not been well-established. That is the focus of this paper.

The Boussinesq one-equation model defined by Eq. (52) in Sec. V is well known to be integrable and allows bidirectional soliton solutions [6], but few people realize that its solutions of head-on collision are not physically meaningful for water waves (see Sec. V of the paper for details).

Weakly nonlinear and weakly dispersive waves on a layer of water with a uniform depth (scaled to be 1) can be modeled by generalized Boussinesq equations [7], in which wave elevation $\zeta$ and velocity vector $\boldsymbol{u}=(u, v)$ are unknown functions of space $(x, y)$ and time $t$. Picking different velocity results in different systems. Depth-mean, bottom, and surface velocities are three popular choices of the velocity variables. The corresponding model equations derived by $\mathrm{Wu}$ and Zhang are as follows [8]. (a) The $\{\zeta, \overline{\boldsymbol{u}}\}$ system: the depth-mean velocity basis

$$
\begin{gathered}
\zeta_{t}+\nabla \cdot[(1+\zeta) \overline{\boldsymbol{u}}]=0, \\
\overline{\boldsymbol{u}}_{t}+\overline{\boldsymbol{u}} \cdot \nabla \overline{\boldsymbol{u}}+\nabla \zeta=\frac{1}{3} \nabla^{2} \overline{\boldsymbol{u}}_{t} .
\end{gathered}
$$

(b) The $\left\{\zeta, \boldsymbol{u}_{0}\right\}$ system: the bottom variable basis

$$
\begin{gathered}
\zeta_{t}+\nabla \cdot\left[(1+\zeta) \boldsymbol{u}_{0}\right]=\frac{1}{6} \nabla \cdot \nabla^{2} \boldsymbol{u}_{0}, \\
\boldsymbol{u}_{0 t}+\boldsymbol{u}_{0} \cdot \nabla \boldsymbol{u}_{0}+\nabla \zeta=\frac{1}{2} \nabla^{2} \boldsymbol{u}_{0 t} .
\end{gathered}
$$

(c) The $\{\zeta, \hat{\boldsymbol{u}}\}$ system: the surface variable basis

$$
\begin{gathered}
\zeta_{t}+\nabla \cdot[(1+\zeta) \hat{\boldsymbol{u}}]=-\frac{1}{3} \nabla \cdot \nabla^{2} \hat{\boldsymbol{u}}, \\
\hat{\boldsymbol{u}}_{t}+\hat{\boldsymbol{u}} \cdot \nabla \hat{\boldsymbol{u}}+\nabla \zeta=0,
\end{gathered}
$$

where $\nabla=\left(\partial_{x}, \partial_{y}\right)$ is a gradient operator, and $\overline{\boldsymbol{u}}, \boldsymbol{u}_{0}$ and $\hat{\boldsymbol{u}}$ are depth-mean, bottom, and surface velocities, respectively. All three systems allow solitary wave solutions and their interactions, such as overtaking, head-on, and oblique collisions. But for some systems, the collisions may not be clean. By clean we mean that the solitary waves do not change their shapes and speeds after the collision and they are not followed by any dispersive tail. The only changes after the interaction are their phase shifts. Whether the collision is clean depends on the integrability of the system. If a system is integrable and has exact solutions, then the interaction of solitary waves will be clean. For clarity, in this paper we define soliton to be a clean interacting solitary wave in an integrable system. Only when the collision is clean and the solitary waves behave like particles during the interaction do we call them solitons. One has to be aware of the difference between the two concepts of solitary wave and soliton. A soliton is a kind of solitary wave, but a solitary wave may not be a soliton depending on whether or not the system is integrable and the interaction is clean.

Given the fact that the three systems are widely used by engineers to study water waves during a coastal and harbor design, the mathematical properties such as integrability and solitary wave (soliton) solutions of the three systems are crucial to us. Ironically our understanding about the integrability of the three systems is so poor that we only know the property of the reduced $(1+1)$ version of the surface-variable system, 


$$
\begin{gathered}
\zeta_{t}+[(1+\zeta) u]_{x}=-\frac{1}{3} u_{x x x}, \\
u_{t}+u u_{x}+\zeta_{x}=0 .
\end{gathered}
$$

This system, thereafter referred to as system (7),(8), has been shown to be integrable and has three Hamiltonian structures $[9,10]$. The integrabilities of the original $(2+1)$ version of $(c)$ and two other systems, (a) and (b), are open questions. A recent paper obtains some interesting and exact solutions of system (c) by using the Painlevé method [11]. In fact, system $(7),(8)$ is shown to be equivalent to the Broer-Kaup (BK) system [10]. In this paper, we show that the system is also equivalent to a member of the Ablowitz-Kaup-Newell-Segur (AKNS) system. Unlike the $\mathrm{KdV}$ equation, system (7),(8) is derived without the assumption that waves propagate in one direction. Since the system is integrable, allows soliton solution, and is bidirectional, it is therefore a natural candidate for modeling bidirectional solitons on water.

With system (7),(8) as our model, we find that its bidirectional soliton solution can be obtained from the Dirac equation, i.e., the AKNS spectral problem. The Schrödinger equation is the first ordinary differential equation that can be used to derive soliton integrable equations [12]; the AKNS system is the second one [13]. But no one has found that a member of the AKNS hierarchy can be used to describe bidirectional solitons on water. There is extensive literature on the study of the solution of system (7),(8) or the BK system [14], but no one has linked the solutions to the bidirectional solitons on water. About 35 years after GGKM, we find that the AKNS system can be used to generate bidirectional solitons on water. The solution agrees with that of the $\mathrm{KdV}$ equation up to the same level of the perturbation scheme for the unidirectional solitons. Existing literature uses two unidirectional solitons from the $\mathrm{KdV}$ equation and an interacting term from the perturbation scheme to describe the head-on interaction [2]. To the best of our knowledge, our solution is the only one that is both exact and physically meaningful for water waves.

In this paper, we study bidirectional solitons on water by using the model of system (7),(8). The properties of a single soliton are presented in Sec. II. In Sec. III, a multisoliton solution is derived by using the Darboux transformation on an equivalent AKNS system. The mechanics of soliton interaction is discussed in Sec. IV. A comparison with the Boussinesq one equation is given in Sec. V. Finally, we conclude the paper in Sec.VI.

\section{SINGLE SOLITON SOLUTION}

We now study the properties of a single soliton, such as shape, mass, and the relationship between the wave speed and the wave amplitude.

The soliton solution can be obtained by setting $\zeta=\zeta(s)$ and $u=u(s)$, where $s=x-\lambda t$ and $\lambda$ is the undetermined wave speed. Substituting these relations into Eqs. (7) and (8) and integrating the resulting equations once under the regularity condition at infinity, we obtain

$$
-\lambda \zeta+(1+\zeta) u=-\frac{1}{3} u_{s s}
$$

$$
-\lambda u+\frac{1}{2} u^{2}+\zeta=0 .
$$

After eliminating $\zeta$ from these equations, integrating once more results in

$$
u_{s}^{2}=\frac{3}{4} u^{2}(2 \lambda+2-u)(2 \lambda-2-u) .
$$

This equation has a solitary wave solution as

$$
u_{B}(s ; \lambda)=\frac{2\left(\lambda^{2}-1\right)}{\lambda+\cosh \sqrt{3\left(\lambda^{2}-1\right)} s},
$$

where the subscript $B$ indicates the solution of the Boussinesq equation. This solution agrees with the one in an implicit form given in Ref. [8]. Substituting the solution of velocity into Eq. (10) gives the wave elevation as

$$
\zeta_{B}(s ; \lambda)=\frac{2\left(\lambda^{2}-1\right)\left[1+\lambda \cosh \sqrt{3\left(\lambda^{2}-1\right)} s\right]}{\left[\lambda+\cosh \sqrt{3\left(\lambda^{2}-1\right)} s\right]^{2}},
$$

hereafter referred to as the Boussinesq soliton. The wave speed $\lambda$ and the wave amplitude $a$ is found from Eq. (13) to have the relationship

$$
\lambda=1+\frac{1}{2} a
$$

Integrating the wave elevation (13) over the whole space domain gives us the mass under the soliton

$$
m_{B}(\lambda)=\int_{-\infty}^{\infty} \zeta_{B}(s ; \lambda) d s=\frac{4}{\sqrt{3}} \sqrt{\lambda^{2}-1}=\frac{4}{\sqrt{3}} \sqrt{(1+a / 4) a}
$$

Differentiating Eq. (13) twice and evaluating at the origin gives us

$$
\zeta_{B}^{\prime \prime}(0 ; \lambda)=-6(2-\lambda)(\lambda-1)^{2}
$$

Therefore, the soliton has a single peak when $\lambda<2$ and double peaks when $\lambda>2$. The soliton appears to have some remarkable features. It is single-peaked when the wave amplitude is not larger than 2, and double-peaked when the wave amplitude is larger than 2. As is well known, the Boussinesq model is only valid for the water waves with small amplitude, i.e., wave amplitude smaller than water depth (scaled to be 1 here). Therefore, the new feature of the double-peaked soliton is not physically meaningful for the water wave.

Under the assumption of unidirectional wave propagation, Eqs. (7) and (8) can be further reduced to the $\mathrm{KdV}$ equation by a perturbation scheme [15],

$$
\zeta_{t}+\zeta_{x}+\frac{3}{2} \zeta \zeta_{x}+\frac{1}{6} \zeta_{x x x}=0,
$$

which has a solitary wave solution

$$
\zeta_{K}(s ; \lambda)=a \operatorname{sech}^{2} \frac{\sqrt{3 a}}{2} s, \quad s=x-\lambda t, \quad \lambda=1+\frac{a}{2},
$$


where the subscript $K$ indicates the $K d V$ soliton. Integrating the wave elevation (18) over the whole space domain gives us the mass under the KdV soliton,

$$
m_{K}(\lambda)=\int_{-\infty}^{\infty} \zeta_{K}(s ; \lambda) d s=\frac{4}{\sqrt{3}} \sqrt{2(\lambda-1)}=\frac{4}{\sqrt{3}} \sqrt{a},
$$

which is slightly smaller than the mass $m_{B}$ under the Boussinesq soliton.

The relations between the wave speed $\lambda$ and the wave amplitude $a$ are the same for both the Boussinesq and KdV solitons, i.e., $\lambda=1+a / 2$. A comparison between the Boussinesq soliton (13) and the KdV soliton of Eq. (18) shows that they agree very well for the waves with small amplitude except that the former is slightly fatter.

\section{MULTISOLITON SOLUTION}

With scaling transformation,

$$
\frac{\sqrt{3}}{2} x \rightarrow x, \frac{\sqrt{3}}{2} t \rightarrow t,
$$

Eqs. (7) and (8) become

$$
\begin{gathered}
\zeta_{t}+[(1+\zeta) u]_{x}=-\frac{1}{4} u_{x x x}, \\
u_{t}+u u_{x}+\zeta_{x}=0 .
\end{gathered}
$$

The Lax pair of the system is

$$
\begin{gathered}
\phi_{x x}=\left(\lambda^{2}+\lambda u+\frac{1}{4} u^{2}-\zeta-1\right) \phi, \\
\phi_{t}=\frac{1}{4} u_{x} \phi+\left(\lambda-\frac{1}{2} u\right) \phi_{x} .
\end{gathered}
$$

By using the transformation

$$
u=-v, \quad \zeta=-1+w-\frac{1}{2} v_{x},
$$

we can convert the system to the BK system,

$$
\begin{gathered}
v_{t}=\frac{1}{2}\left(v^{2}+2 w-v_{x}\right)_{x}, \\
w_{t}=\left(v w+\frac{1}{2} w_{x}\right)_{x} .
\end{gathered}
$$

In Ref. [16], we solve the BK system by using the Darboux transformation and obtain a multisoliton solution. But the result produced with a recursive algorithm is very lengthy for interaction among a large number of solitons.
Here we adopt a new approach and convert the system to a member of the AKNS system and obtain a much cleaner version of the multisoliton solution.

Introducing the transformation

$$
q=e^{\int u d x}, \quad r=-\left(1+\zeta-\frac{1}{2} u_{x}\right) e^{-\int u d x}
$$

or

$$
u=\frac{q_{x}}{q}, \quad \zeta=-1-q r+\frac{1}{2} u_{x}
$$

we have an equivalent system for $q$ and $r$,

$$
\begin{aligned}
& q_{t}+\frac{1}{2} q_{x x}-q^{2} r-q=0, \\
& r_{t}-\frac{1}{2} r_{x x}+q r^{2}+r=0,
\end{aligned}
$$

which is a member of the AKNS hierarchy. It is well known that the Lax pair of the system is

$$
\begin{aligned}
& \psi_{x}=M \psi, \quad \psi=\left(\psi_{1}, \psi_{2}\right)^{T}, \quad M=\left(\begin{array}{cc}
-\lambda & q \\
r & \lambda
\end{array}\right), \\
& \psi_{t}=N \psi, \quad N=\left(\begin{array}{cc}
-\lambda^{2}+\frac{1}{2} q r+\frac{1}{2} & \lambda q-\frac{1}{2} q_{x} \\
\lambda r+\frac{1}{2} r_{x} & \lambda^{2}-\frac{1}{2} q r-\frac{1}{2}
\end{array}\right) .
\end{aligned}
$$

The Darboux transformation on the AKNS system given in Ref. [17] is as follows. Let

$$
\phi^{\prime}=T \phi, \quad T=\lambda^{n} I+\sum_{j=1}^{n} T_{j} \lambda^{n-j}, \quad T_{j}=\left(\begin{array}{ll}
a_{2 j-1} & a_{2 j} \\
b_{2 j-1} & b_{2 j}
\end{array}\right),
$$

where $I$ is a $2 \times 2$ identity matrix and $\phi$ is a solution of Eqs. (29) and (30); then $\phi^{\prime}$ is a solution of the equation

$$
\phi_{x}^{\prime}=M^{\prime} \phi^{\prime}, \quad \phi_{t}^{\prime}=N^{\prime} \phi^{\prime},
$$

where $M^{\prime}$ and $N^{\prime}$ are the same as $M$ and $N$ in Eqs. (29) and (30), but with $q, r, q_{x}$, and $r_{x}$ replaced by $q^{\prime}, r^{\prime}, q_{x}^{\prime}$, and $r_{x}^{\prime}$.

We assume $\lambda_{i} \neq \lambda_{j}$ for $i \neq j, i=1,2, \ldots, 2 n$, and denote

$$
\phi_{1, j}=\phi_{1}\left(x, \lambda_{j}\right), \quad \phi_{2, j}=\phi_{2}\left(x, \lambda_{j}\right) .
$$

We define a $2 n \times 2 n$ matrix $H$ to be the following:

$$
H=\left(\begin{array}{ccccccc}
\lambda_{1}^{n-1} \phi_{1,1} & \lambda_{1}^{n-1} \phi_{2,1} & \lambda_{1}^{n-2} \phi_{1,1} & \lambda_{1}^{n-2} \phi_{2,1} & \cdots & \phi_{1,1} & \phi_{2,1} \\
\lambda_{2}^{n-1} \phi_{1,2} & \lambda_{2}^{n-1} \phi_{2,2} & \lambda_{2}^{n-2} \phi_{1,2} & \lambda_{2}^{n-2} \phi_{2,2} & \cdots & \phi_{1,2} & \phi_{2,2} \\
\vdots & \vdots & \vdots & \vdots & \cdots & \vdots & \vdots \\
\lambda_{2 n}^{n-1} \phi_{1,2 n} & \lambda_{2 n}^{n-1} \phi_{2,2 n} & \lambda_{2 n}^{n-2} \phi_{1,2 n} & \lambda_{2 n}^{n-2} \phi_{2,2 n} & \cdots & \phi_{1,2 n} & \phi_{2,2 n}
\end{array}\right) .
$$


Solving the equations

$$
\begin{aligned}
& H\left(\begin{array}{c}
a_{1} \\
a_{2} \\
\vdots \\
a_{2 n}
\end{array}\right)=\left(\begin{array}{c}
-\lambda_{1}^{n} \phi_{1,1} \\
-\lambda_{2}^{n} \phi_{1,2} \\
\vdots \\
-\lambda_{2 n}^{n} \phi_{1,2 n}
\end{array}\right) \equiv A, \\
& H\left(\begin{array}{c}
b_{1} \\
b_{2} \\
\vdots \\
b_{2 n}
\end{array}\right)=\left(\begin{array}{c}
-\lambda_{1}^{n} \phi_{2,1} \\
-\lambda_{2}^{n} \phi_{2,2} \\
\vdots \\
-\lambda_{2 n}^{n} \phi_{2,2 n}
\end{array}\right) \equiv B
\end{aligned}
$$

gives us $a_{i}$ and $b_{i}, i=1,2,3, \ldots, 2 n$. Then

$$
q^{\prime}=q+2 a_{2}, \quad r^{\prime}=r-2 b_{1},
$$

where

$$
a_{2}=\frac{\operatorname{det} H_{2}}{\operatorname{det} H}, \quad b_{1}=\frac{\operatorname{det} H_{1}}{\operatorname{det} H}
$$

and $H_{2}$ is a $2 n \times 2 n$ matrix of $H$ with the second column replaced by $A$, and $H_{1}$ is a $2 n \times 2 n$ matrix of $H$ with the first column replaced by $B$.

For a layer of quiescent water without any waves, wave elevation is $\zeta=0$ and velocity is $u=0$, and correspondingly $q=1$ and $r=-1$. Therefore, we take $(q, r)=(1,-1)$ as our initial seed to implement the Darboux transformation. With this initial seed, we have the following two sets of basic solutions for the spectral problem (29),(30):

$$
\begin{aligned}
\phi_{1, j}= & \cosh \xi_{j}, \quad \phi_{2, j}=c_{j} \sinh \xi_{j}+\lambda_{j} \cosh \xi_{j}, \\
& j \text { is an odd number, } \\
\phi_{1, j}= & \sinh \xi_{j}, \quad \phi_{2, j}=c_{j} \cosh \xi_{j}+\lambda_{j} \sinh \xi_{j},
\end{aligned}
$$

$j$ is an even number,

where $\xi_{j}=c_{j}\left(x+\lambda_{j} t\right)$ and $c_{j}=\sqrt{\lambda_{j}^{2}-1}$. The eigenvalue $\lambda_{j}$ is the wave speed of a soliton. The soliton is right-going if $\lambda_{j}$ $<-1$ and left-going if $\lambda_{j}>1$. For a single left-going soliton, its amplitude $a$ and speed $\lambda$ satisfy the relationship given by Eq. (14).

We now construct a multisoliton solution with $2 \mathrm{~m}$ leftgoing and $2 l$ right-going solitons. The power of the eigenvalue in the Darboux transformation is taken to be $n=m$ $+l$. First we rank the solitons by their amplitudes (or speeds). For the $2 m$ left-going solitons, we assume $\lambda_{2 m}$ $>\lambda_{2 m-1}>\cdots>\lambda_{1}>1$. For the $2 l$ right-going solitons, we assume $\lambda_{2 l}^{*}<\lambda_{2 l-1}^{*}<\cdots<\lambda_{1}^{*}<-1$. With the eigenfunctions defined in Eqs. (37),(38) for both $\lambda_{j}$ and $\lambda_{j}^{*}$, we can obtain the soliton solution as follows:

$$
u=\frac{q_{x}^{\prime}}{q^{\prime}}, \quad \zeta=-1-q^{\prime} r^{\prime}+\frac{1}{2} u_{x},
$$

where

$$
q^{\prime}=1+2 a_{2}, \quad r^{\prime}=-1-2 b_{1} .
$$

$a_{2}$ and $b_{1}$ are defined by Eq. (36).

This is the solution for the interaction of an even number of solitons in both directions. To obtain an odd number of solitons, we can simply set the first eigenvalue to be 1 for a left-going soliton and -1 for a right-going soliton. In other words, an odd number of soliton solution can be treated as an even number of solitons in which one of the solitons has zero amplitude.

The Darboux transformation provided in Ref. [16] for the BK system is of the first order of the eigenvalue. It can be used to generate a two-soliton solution. For a $2(m+l)$-soliton solution, the Darboux transformation has to be applied $m+l$ times. The result would be very messy. Here due to a nice property of the AKNS system, we only need to apply the Darboux transformation once to generate a solution with an arbitrary number of solitons. It only involves an evaluation of the determinant of a $2(m+l) \times 2(m+l)$ matrix. The result of a multisoliton solution is much cleaner than that produced with the Darboux transformation of the BK system [16].

\section{MECHANICS OF SOLITON INTERACTION}

For a single right-going soliton solution, we can take $m$ $=0$ and $l=1$ with the following eigenvalues and eigenfunctions:

$$
\begin{gathered}
\lambda_{1}^{*}=-1, \quad \phi_{1,1}=1, \quad \phi_{2,1}=-1 \\
\lambda_{2}^{*}=-\lambda<-1, \quad \phi_{1,2}=\sinh \xi, \quad \phi_{2,2}=c \cosh \xi-\lambda \sinh \xi
\end{gathered}
$$

With some algebra, one may easily verify that the solution given by Eq. (39) is identical to that obtained with direct integration given by Eqs. (12),(13).

\section{A. Overtaking collision}

For a solution with two solitons overtaking collision, we take $m=0$ and $l=1$ with the following eigenvalues and eigenfunctions:

$$
\begin{gathered}
\lambda_{1}^{*}=-\lambda_{1}<-1, \quad \phi_{1,1}=\cosh \xi_{1}, \\
\phi_{2,1}=c_{1} \sinh \xi_{1}-\lambda_{1} \cosh \xi_{1}, \\
\lambda_{2}^{*}=-\lambda_{2}<-\lambda_{1}, \quad \phi_{1,2}=\sinh \xi_{2}, \\
\phi_{2,2}=c_{2} \cosh \xi_{2}-\lambda_{2} \sinh \xi_{2},
\end{gathered}
$$

where $\lambda_{1}$ and $\lambda_{2}$ are two positive numbers. The solution to system (7),(8), given by Eq. (39), can be written in a closed form as 


$$
\begin{gathered}
u=\frac{2\left(\lambda_{2}-\lambda_{1}\right)\left[c_{2}^{2}-c_{1}^{2} \tanh ^{2} \xi_{1} \tanh ^{2} \xi_{2}-\left(\lambda_{2}^{2}-\lambda_{1}^{2}\right) \tanh ^{2} \xi_{2}\right]}{\left(c_{2}-c_{1} \tanh \xi_{1} \tanh \xi_{2}\right)^{2}-\left(\lambda_{2}-\lambda_{1}\right)^{2} \tanh ^{2} \xi_{2}}, \\
\zeta=-1+\frac{c_{2}-c_{1} \tanh \xi_{1} \tanh \xi_{2}+\left(\lambda_{2}-\lambda_{1}\right) \tanh \xi_{2}}{c_{2}-c_{1} \tanh \xi_{1} \tanh \xi_{2}-\left(\lambda_{2}-\lambda_{1}\right) \tanh \xi_{2}}\left[1-2 \frac{\left(\lambda_{2}-\lambda_{1}\right)\left(c_{1} \tanh \xi_{1}-\lambda_{1}\right)\left(c_{2}-\lambda_{2} \tanh \xi_{2}\right)}{c_{2}-c_{1} \tanh \xi_{1} \tanh \xi_{2}-\left(\lambda_{2}-\lambda_{1}\right) \tanh \xi_{2}}\right]+\frac{1}{\sqrt{3}} u_{x}, \\
\xi_{i}=\frac{\sqrt{3}}{2} c_{i}\left(x-\lambda_{i} t\right), \quad c_{i}=\sqrt{\lambda_{i}^{2}-1}, \quad i=1,2, \quad \lambda_{2}>\lambda_{1}>1,
\end{gathered}
$$

where $x$ and $t$ have been converted to the original coordinates before the scaling transformation (20), $\lambda_{1}$ and $\lambda_{2}$ are the speeds of the two solitons, with $\lambda_{2}$ larger than $\lambda_{1}$. The soliton with the speed $\lambda_{2}$ is taking over the soliton with the speed $\lambda_{1}$. The process of overtaking interaction can be easily seen with the asymptotic limit of the solution (40),(41): as $t \rightarrow-\infty$,

$$
\begin{aligned}
& \zeta(x, t) \rightarrow \zeta_{B}\left(x-\lambda_{1} t-\Delta_{1} ; \lambda_{1}\right)+\zeta_{B}\left(x-\lambda_{2} t+\Delta_{2} ; \lambda_{2}\right), \\
& u(x, t) \rightarrow u_{B}\left(x-\lambda_{1} t-\Delta_{1} ; \lambda_{1}\right)+u_{B}\left(x-\lambda_{2} t+\Delta_{2} ; \lambda_{2}\right),
\end{aligned}
$$

and as $t \rightarrow+\infty$,

$$
\begin{aligned}
& \zeta(x, t) \rightarrow \zeta_{B}\left(x-\lambda_{1} t+\Delta_{1} ; \lambda_{1}\right)+\zeta_{B}\left(x-\lambda_{2} t-\Delta_{2} ; \lambda_{2}\right), \\
& u(x, t) \rightarrow u_{B}\left(x-\lambda_{1} t+\Delta_{1} ; \lambda_{1}\right)+u_{B}\left(x-\lambda_{2} t-\Delta_{2} ; \lambda_{2}\right),
\end{aligned}
$$

where $\zeta_{B}(s ; \lambda)$ and $u_{B}(s ; \lambda)$ are the wave elevation and surface velocity of the single-soliton solution given by Eqs. (13),(12), and the total phase shift of the two solitons is given by the following:

$$
\begin{aligned}
& 2 \Delta_{1}=\frac{2}{\sqrt{3\left(\lambda_{1}^{2}-1\right)}} \operatorname{arccosh} \frac{\lambda_{1} \lambda_{2}-1}{\lambda_{2}-\lambda_{1}}, \\
& 2 \Delta_{2}=\frac{2}{\sqrt{3\left(\lambda_{2}^{2}-1\right)}} \operatorname{arccosh} \frac{\lambda_{1} \lambda_{2}-1}{\lambda_{2}-\lambda_{1}} .
\end{aligned}
$$

Since the mass has been obtained previously in Eq. (15) as

$$
m_{i}=\frac{4}{\sqrt{3}} \sqrt{\lambda_{i}^{2}-1}, \quad i=1,2
$$

the conservation of momentum can be easily verified by

$$
2 m_{1} \Delta_{1}=2 m_{2} \Delta_{2}=\frac{8}{3} \quad \operatorname{arccosh} \frac{\lambda_{1} \lambda_{2}-1}{\lambda_{2}-\lambda_{1}} .
$$

The asymptotic limit of the solution shows that it is symmetric about the origin. To study the wave properties near the center of an encounter, we find with some algebra that the wave elevation and its first and second derivatives at the origin are

$$
\begin{gathered}
\zeta(0,0)=\left(1+\frac{1}{2} a_{1}\right)\left(a_{2}-a_{1}\right), \\
\zeta_{x}(0,0)=0, \\
\zeta_{x x}(0,0)=-\frac{3}{16}\left(a_{2}-a_{1}\right)\left[8\left(a_{2}-3 a_{1}\right)-4\left(a_{2}^{2}-7 a_{2} a_{1}+9 a_{1}^{2}\right)\right. \\
\left.-a_{1}\left(2 a_{2}^{2}-9 a_{2} a_{1}+9 a_{1}^{2}\right)\right],
\end{gathered}
$$

where $a_{i}=2\left(\lambda_{i}-1\right), i=1,2$ are the wave amplitudes of two solitons. Solving $\zeta_{x x}(0,0)=0$, i.e.,

$$
\frac{a_{2}}{a_{1}}=3+\frac{1}{2}\left(a_{2} \frac{a_{2}}{a_{1}}-7 a_{2}+9 a_{1}\right)+\frac{1}{8}\left(2 a_{2}^{2}-9 a_{2} a_{1}+9 a_{1}^{2}\right),
$$

we find the critical amplitude ratio $R_{c}$ is very close to 3 for the small amplitude waves. The wave elevation at $t=0$ has a single peak if $a_{2} / a_{1}<R_{c}$ and two peaks if $a_{2} / a_{1}>R_{c}$.

The overtaking collision can also be modeled by the KdV equation (17). The solution can be obtained by applying Hirota's method [6]; see, e.g., Ref. [2]. In the exact solution of the $\mathrm{KdV}$ equation, the phase shift of soliton 2 is given by

$$
2 \Delta_{2}=\frac{2}{\sqrt{3 a_{2}}} \ln \frac{\left(\sqrt{a_{2}}+\sqrt{a_{1}}\right)^{2}}{a_{2}-a_{1}} .
$$

One may easily show that the phase shift agrees to the leading order with that in Eq. (42) given by our exact solution of the Boussinesq equation. For the wave profile at $t=0$, the critical amplitude ratio, derived in Ref. [2], is equal to 3 for the solution of the $\mathrm{KdV}$ equation, which also agrees to the leading order with our result in Eq. (43).

\section{B. Head-on collision}

For a solution with two-soliton head-on collision, we take $m=0$ and $l=1$ with the following eigenvalues and eigenfunctions: 


$$
\begin{gathered}
\lambda_{1}^{*}=-\lambda_{1}<-1, \quad \phi_{1,1}=\cosh \xi_{1}, \quad \phi_{2,1}=c_{1} \sinh \xi_{1}-\lambda_{1} \cosh \xi_{1}, \\
\lambda_{2}^{*}=\lambda_{2}>1, \quad \phi_{1,2}=\cosh \xi_{2}, \quad \phi_{2,2}=c_{2} \sinh \xi_{2}+\lambda_{2} \cosh \xi_{2} .
\end{gathered}
$$

The solution of system (7), (8) given by Eq. (39) can be written in a closed form as follows:

$$
\begin{gathered}
u=\frac{2\left(\lambda_{1}+\lambda_{2}\right)\left(\lambda_{2}^{2}-\lambda_{1}^{2}-c_{2}^{2} \tanh ^{2} \xi_{2}+c_{1}^{2} \tanh ^{2} \xi_{1}\right)}{\left(c_{2} \tanh \xi_{2}-c_{1} \tanh \xi_{1}\right)^{2}-\left(\lambda_{1}+\lambda_{2}\right)^{2}}, \\
\zeta=-1+\frac{c_{2} \tanh \xi_{2}-c_{1} \tanh \xi_{1}-\lambda_{1}-\lambda_{2}}{c_{2} \tanh \xi_{2}-c_{1} \tanh \xi_{1}+\lambda_{1}+\lambda_{2}}\left[1+2 \frac{\left(\lambda_{1}+\lambda_{2}\right)\left(c_{1} \tanh \xi_{1}-\lambda_{1}\right)\left(c_{2} \tanh \xi_{2}+\lambda_{2}\right)}{c_{2} \tanh \xi_{2}-c_{1} \tanh \xi_{1}+\lambda_{1}+\lambda_{2}}\right]+\frac{1}{\sqrt{3}} u_{x}, \\
\xi_{1}=\frac{\sqrt{3}}{2} c_{1}\left(x-\lambda_{1} t\right), \quad \xi_{2}=\frac{\sqrt{3}}{2} c_{2}\left(x+\lambda_{2} t\right), \quad c_{i}=\sqrt{\lambda_{i}^{2}-1}, \quad \lambda_{i}>1, \quad i=1,2,
\end{gathered}
$$

where $x$ and $t$ are the original coordinates before the scaling transformation. The soliton with speed $\lambda_{1}$ is moving from the left to the right. The soliton with speed $\lambda_{2}$ is moving from the right to the left. At $t=0$, the two solitons merge into a single peak. One may verify that $\zeta_{x}(0,0)=0$, i.e., the solution is symmetric about the origin. Therefore, the maximum amplitude appears at the origin, i.e.,

$$
\zeta_{\max }=\zeta(0,0)=a_{1}+a_{2}+\frac{1}{2} a_{1} a_{2}
$$

which agrees to the leading order with the solution obtained by using the perturbation method in Refs. [1,2]. For the head-on collision of two solitons with the same amplitude, $a_{1}=a_{2}=a$, the wave elevation at $t=0$ can be simplified and given by

$$
\zeta(x, 0)=\left(2 a+\frac{1}{2} a^{2}\right) \operatorname{sech}^{2}\left[\frac{1}{4} \sqrt{3 a(4+a)} x\right],
$$

and the velocity at $t=0$ is zero for all $x$.

After the head-on collision, each soliton experiences a backward phase shift. The asymptotic analysis of the solution (45), (46) leads to the following limits: as $t \rightarrow-\infty$,

$$
\begin{aligned}
& \zeta(x, t) \rightarrow \zeta_{B}\left(x-\lambda_{1} t-\Delta_{1} ; \lambda_{1}\right)+\zeta_{B}\left(x+\lambda_{2} t+\Delta_{2} ; \lambda_{2}\right), \\
& u(x, t) \rightarrow u_{B}\left(x-\lambda_{1} t-\Delta_{1} ; \lambda_{1}\right)-u_{B}\left(x+\lambda_{2} t+\Delta_{2} ; \lambda_{2}\right),
\end{aligned}
$$

and as $t \rightarrow+\infty$,

$$
\begin{aligned}
& \zeta(x, t) \rightarrow \zeta_{B}\left(x-\lambda_{1} t+\Delta_{1} ; \lambda_{1}\right)+\zeta_{B}\left(x+\lambda_{2} t-\Delta_{2} ; \lambda_{2}\right), \\
& u(x, t) \rightarrow u_{B}\left(x-\lambda_{1} t+\Delta_{1} ; \lambda_{1}\right)-u_{B}\left(x+\lambda_{2} t-\Delta_{2} ; \lambda_{2}\right),
\end{aligned}
$$

where $\zeta_{B}(s ; \lambda)$ and $u_{B}(s ; \lambda)$ are the wave elevation and surface velocity of the single-soliton solution given by Eqs. (13),(12), and the total phase shift of the two solitons is given by

$$
\begin{aligned}
& 2 \Delta_{1}=\frac{2}{\sqrt{3\left(\lambda_{1}^{2}-1\right)}} \operatorname{arccosh} \frac{\lambda_{1} \lambda_{2}+1}{\lambda_{1}+\lambda_{2}}, \\
& 2 \Delta_{2}=\frac{2}{\sqrt{3\left(\lambda_{2}^{2}-1\right)}} \operatorname{arccosh} \frac{\lambda_{1} \lambda_{2}+1}{\lambda_{1}+\lambda_{2}} .
\end{aligned}
$$

The conservation of momentum can be easily verified by

$$
2 m_{1} \Delta_{1}=2 m_{2} \Delta_{2}=\frac{8}{3} \operatorname{arccosh} \frac{\lambda_{1} \lambda_{2}+1}{\lambda_{1}+\lambda_{2}} .
$$

If we expand the phase shift for small amplitude waves, i.e., $a_{i} \ll 1, i=1,2$, we will have the leading-order term,

$$
2 \Delta_{1} \simeq \frac{1}{\sqrt{3}} \sqrt{a_{2}}, \quad 2 \Delta_{2} \simeq \frac{1}{\sqrt{3}} \sqrt{a_{1}},
$$

which again agrees with the result obtained with the perturbation approach.

\section{COMPARISON WITH THE BOUSSINESQ ONE EQUATION}

In addition to system (7),(8), the Boussinesq one equation

$$
\zeta_{t t}-\zeta_{x x}-\frac{3}{2}\left(\zeta^{2}\right)_{x x}-\frac{1}{3} \zeta_{x x x x}=0
$$

is often used in the literature to model bidirectional solitons, but the result of the head-on collision is not physically meaningful for water waves. The equation is integrable and has two Hamiltonian structures, see, e.g., Ref. [18]. Its single soliton solution 


$$
\zeta(x, t)=a \operatorname{sech}^{2} \frac{\sqrt{3 a}}{2}(x-\lambda t), \quad \lambda=\sqrt{1+a}<1+\frac{a}{2}
$$

has the same shape as that of the KdV soliton with the same amplitude, but travels at a slightly slower speed than the $\mathrm{KdV}$ soliton. Its multisoliton solution has been provided by Hirota [6]. For example, the solution of two soliton head-on collision is given as

$$
\begin{gathered}
\zeta(x, t)=4 \frac{k_{1}^{2} \operatorname{sech}^{2} \xi_{1}+k_{2}^{2} \operatorname{sech}^{2} \xi_{2}+C \operatorname{sech}^{2} \xi_{1} \operatorname{sech}^{2} \xi_{2}}{\left[\cosh (\theta / 2)+\sinh (\theta / 2) \tanh \xi_{1} \tanh \xi_{2}\right]^{2}}, \\
\xi_{1}=\sqrt{3} k_{1}\left(x-\lambda_{1}\right) t, \quad \xi_{2}=\sqrt{3} k_{2}\left(x+\lambda_{2} t\right),
\end{gathered}
$$

$$
\begin{gathered}
C=\sinh (\theta / 2)\left[\left(k_{1}^{2}+k_{2}^{2}\right) \sinh (\theta / 2)+2 k_{1} k_{2} \cosh (\theta / 2)\right], \\
\theta=\frac{1}{2} \ln \frac{\left(\lambda_{1}+\lambda_{2}\right)^{2}+12\left(k_{1}-k_{2}\right)^{2}}{\left(\lambda_{1}+\lambda_{2}\right)^{2}+12\left(k_{1}+k_{2}\right)^{2}}, \\
\lambda_{1}=\sqrt{1+a_{1}}, \quad \lambda_{2}=\sqrt{1+a_{2}}, \quad k_{1}=\frac{1}{2} \sqrt{a_{1}}, \quad k_{2}=\frac{1}{2} \sqrt{a_{2}},
\end{gathered}
$$

where $a_{1}$ and $a_{2}$ are the amplitudes of the two solitons. The soliton with amplitude $a_{1}$ is right-going. The soliton with amplitude $a_{2}$ is left-going. One may verify that $\zeta_{x}(0,0)=0$, therefore the maximum amplitude during the head-on collision appears at the origin, i.e.,

$$
\begin{aligned}
\zeta_{\max }=\zeta(0,0) & =a_{1}+a_{2}+2 \sqrt{a_{1} a_{2}} \tanh \left(\frac{1}{4} \ln \frac{1+2 a_{1}-3 \sqrt{a_{1} a_{2}}+2 a_{2}+\sqrt{\left(1+a_{1}\right)\left(1+a_{2}\right)}}{1+2 a_{1}+3 \sqrt{a_{1} a_{2}}+2 a_{2}+\sqrt{\left(1+a_{1}\right)\left(1+a_{2}\right)}}\right), \\
& =a_{1}+a_{2}-\frac{3}{2} a_{1} a_{2}+O\left(\alpha^{3}\right) \quad \text { for } a_{1}=O(\alpha), \quad a_{2}=O(\alpha) .
\end{aligned}
$$

The result is smaller than the physically correct result given by Eq. (47). To understand this, one may treat $a_{1}$ and $a_{2}$ as the contribution from the potential energy of each soliton and the joint term $a_{1} a_{2}$ as the contribution from the kineticenergy of the two solitons. The kinetic-energy part of Eq. (53) is negative. This is not physical for water waves. Due to the symmetry, the reflection of a solitary wave on a vertical wall can be treated as the head-on collision of two solitary waves with the same amplitude. Since the Boussinesq one equation (52) cannot be used to physically describe the process of the head-on interaction, it cannot be used to describe the process of run-up of a soliton on a vertical wall.

We now explain the difference between the two models (7),(8) and (52). Introducing velocity potential $\varphi$ that is defined by $u=\varphi_{x}$, we integrate Eq. (8) to obtain $\zeta$ as

$$
\zeta=-\varphi_{t}-\frac{1}{2} \varphi_{x}^{2} .
$$

Substituting the above into Eq. (7) yields an integrable equation for $\varphi$,

$$
\varphi_{t t}-\varphi_{x x}+\frac{1}{2}\left(\varphi_{x}^{2}\right)_{t}+\left(\varphi_{t} \varphi_{x}\right)_{x}+\frac{1}{2}\left(\varphi_{x}^{3}\right)_{x}-\frac{1}{3} \varphi_{x x x x}=0 .
$$

Differentiating with respect to $t$ and substituting Eq. (54) into the equation gives us

$$
\begin{aligned}
\zeta_{t t}- & \zeta_{x x}-\frac{1}{2}\left(\varphi_{x}^{2}\right)_{x x}-\left(\varphi_{t} \varphi_{x}\right)_{x t}-\frac{1}{3} \zeta_{x x x x}=\frac{1}{2}\left(\varphi_{x}^{3}\right)_{x t} \\
& +\frac{1}{6}\left(\varphi_{x}^{2}\right)_{x x x x} .
\end{aligned}
$$

For weakly nonlinear and weakly dispersive water waves, according to the perturbation scheme in Ref. [15], the order of the magnitude of different variables and differentiations is

$$
\zeta=O\left(\epsilon^{2}\right), \quad u=O\left(\epsilon^{2}\right), \quad \partial_{x}=O(\epsilon), \quad \partial_{t}=O(\epsilon),
$$

where $\epsilon$ is the ratio between the water depth and the wavelength. Since $u=\varphi_{x}$, the magnitude of the velocity potential is $\varphi=O(\epsilon)$. Assuming the wave is propagating in one direction, i.e., $\partial_{t}= \pm \partial_{x}+o(\epsilon)$, then the middle two terms in Eq. (55) can be approximated by

$$
\left(\varphi_{x}^{2}\right)_{x x}=\left(\varphi_{t}^{2}\right)_{x x}+o\left(\epsilon^{6}\right), \quad\left(\varphi_{t} \varphi_{x}\right)_{x t}=\left(\varphi_{t}^{2}\right)_{x x}+o\left(\epsilon^{6}\right) .
$$

With the unidirectional assumption, Eq. (55) can be written as

$$
\zeta_{t t}-\zeta_{x x}-\frac{3}{2}\left(\varphi_{t}^{2}\right)_{x x}-\frac{1}{3} \zeta_{x x x x}=o\left(\epsilon^{6}\right) .
$$

Substituting Eq. (54) into the equation yields

$$
\zeta_{t t}-\zeta_{x x}-\frac{3}{2}\left(\zeta^{2}\right)_{x x}-\frac{1}{3} \zeta_{x x x x}=o\left(\epsilon^{6}\right) .
$$

Neglecting the higher-order term at the right-hand side gives Boussinesq one-equation model. The fact that the Boussinesq one equation cannot be used to physically describe the head-on collision of solitons has been pointed out before in the fluid mechanics literature $[19,2]$, but the details of the problem, such as the maximum amplitude during the head-on collision, have not been presented before.

In order to model bidirectional solitons on water, a system has to be integrable and physically correct in both overtaking and head-on collisions at least to the leading order of the perturbation, i.e., to the $\mathrm{KdV}$ level. It seems to us that system (7),(8) is the only serious candidate. It is a natural extension of the $\mathrm{KdV}$ equation from unidirectional solitons to bidirectional solitons. Since system (c) is a physical extension of system (7),(8) from one-space dimension to two-space dimensions, one may have a chance to use system (c) to describe oblique interaction of solitons. Its integrability and exact solutions are interesting topics for further research. 


\section{CONCLUSION}

We study bidirectional solitons on a uniform layer of shallow water. By applying the Darboux transformation method, we find the exact solutions of the Boussinesq two-equation model with wave elevation and surface fluid velocity as two dependent variables. We present the solutions of singlesoliton and multisoliton interactions. The results agree with the $\mathrm{KdV}$ solution for overtaking collision and the perturbation solution for head-on collision. We compare our result with that of the Boussinesq one-equation model and show why the latter does not give a physically correct result on head-on collision. Our bidirectional soliton solution is the only one that is both exact and physically meaningful for water waves to the leading $(\mathrm{KdV})$ level of the perturbation scheme. Our study shows that the Boussinesq surfacevariable equation deserves special attention in the study of bidirectional and obliquely interacting solitons on water.

\section{ACKNOWLEDGMENTS}

We wish to thank Theodore $\mathrm{Y}$. Wu for encouragement. The work of J.E.Z was partially supported by the Research Grants Council of Hong Kong. The work of Y.S.L was supported by the National Basic Research Project: Nonlinear Science, and by the Ministry of Education of China.
[1] C. H. Su and R. M. Mirie, J. Fluid Mech. 98, 509 (1980).

[2] T. Y. Wu, Acta Mech. Sin. 11, 4 (1995).

[3] J. E. Zhang, Ph.D. thesis, Caltech, 1996; J. E. Zhang and T. Y. Wu, J. Eng. Mech. 125, 812 (1999); J. E. Zhang, T. Y. Wu, and T. Y. Hou, Adv. Appl. Mech. 37, 89 (2000).

[4] N. J. Zabusky and M. D. Kruskal, Phys. Rev. Lett. 15, 240 (1965).

[5] C. S. Gardner, J. M. Greene, M. D. Kruskal, and R. M. Miura, Phys. Rev. Lett. 19, 1095 (1967).

[6] R. Hirota, J. Math. Phys. 14, 810 (1973).

[7] T. Y. Wu, Tsunamis-Proceedings of The National Science Foundation Workshop (Tetra Tech, Inc., Pasadena, CA, 1979); T. Y. Wu, J. Eng. Mech. 107, 501 (1981).

[8] T. Y. Wu and J. E. Zhang, in Mathematics is for Solving Problems, edited by L.P. Cook, V. Roytburd, and M. Tulin (SIAM, Philadelphia, 1996), pp. 223-249.

[9] D. J. Kaup, Prog. Theor. Phys. 54, 396 (1975).

[10] B. A. Kupershmidt, Commun. Math. Phys. 99, 51 (1985).

[11] C.-L. Chen, X.-Y. Tang, and S.-Y. Lou, Phys. Rev. E 66, 036605 (2002)

[12] P. D. Lax, Commun. Pure Appl. Math. 21, 467 (1968).

[13] M. J. Ablowitz, D. J. Kaup, A. C. Newell, and H. Segur, Phys.
Rev. Lett. 31, 125 (1973); V. E. Zakharov and A. B. Shabat, Sov. Phys. JETP 34, 62 (1972).

[14] See, for example, R. Conte, M. Musette, and A. Pickering, J. Phys. A 28, 179 (1995); G. A. El, R. H. J. Grimshaw, and M. V. Pavlov, Stud. Appl. Math. 106, 157 (2001); L. J. F. Broer, Appl. Sci. Res. 31, 377 (1975); D. J. Kaup, Prog. Theor. Phys. 54, 72 (1975); V. B. Matveev and M. I. Yavor, Ann. Inst. Henri Poincare, Sect. A 31, 25 (1979); R. L. Sachs, Physica D 30, 1 (1988); R. A. Leo, G. Mancarelia, and G. Soliani, J. Phys. Soc. Jpn. 57, 753 (1988); R. Conte, M. Musette, and A. Pickering, J. Phys. A 27, 2831 (1994); R. Hirota, J. Phys. Soc. Jpn. 54, 2409 (1985); J. Satsuma, K. Kajiwara, J. Matsukidaira, and J. Hietarinta, ibid. 61, 3096 (1992).

[15] T. Y. Wu, Methods Appl. Anal. 1, 108 (1994).

[16] Y.-S. Li, W.-X. Ma, and J. E. Zhang, Phys. Lett. A 275, 60 (2000); Y.-S. Li and J. E. Zhang, ibid. 284, 253 (2001); Y.-S. Li and J. E. Zhang, Chaos, Solitons Fractals 16, 271 (2003).

[17] V. B. Matveev and M. A. Salle, Darboux Transformation and Solitons (Springer-Verlag, Berlin, 1991).

[18] P. J. Olver, Applications of Lie Groups to Differential Equations (Springer-Verlag, New York, 1993).

[19] J. W. Miles, J. Fluid Mech. 106, 131 (1981). 